\title{
A novel function of IMPA2, plays a tumor-promoting role in cervical cancer
}

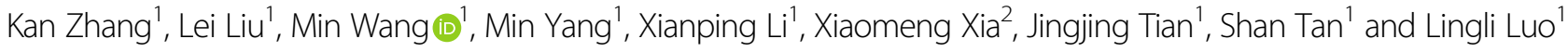

\begin{abstract}
Discovery of genes and molecular mechanism involved in cervical cancer development would promote the prevention and treatment. By comparing gene expression profiles of cervical carcinoma in situ (CCIS) and adjacent normal tissues, we identified a potential cancer-promoting gene, IMPA2. This study aimed to elucidate the role of IMPA2 and underlying molecular mechanisms in cervical cancer progression. To do this expression of IMPA2 was compared between human cervical cancer and corresponding adjacent normal cervical tissues firstly. CCK-8 assay, clone formation assay, wound healing assay, transwell assay, and tumor formation in nude mice were performed to demonstrate the effect of IMPA2 in cervical cancer proliferation and metastasis. Further proteomic profiling and western blotting explored the molecular pathway involved in the IMPA2-regulating process. The results showed that IMPA2 gene expression was upregulated in cervical cancer. Consistently, silencing of IMPA2 suppressed tumor formation in BALB/c nude mice. Short hairpin RNA (shRNA)-mediated IMPA2 silencing significantly inhibited proliferation and colony-forming abilities of cervical cancer cells, while IMPA2 overexpression had little impact. Also, IMPA2 silencing suppressed cellular migration, but overexpression promoted migration. Proteomics analysis revealed the involvement of mitogen-activated protein kinase (MAPK) pathway in tumor-promoting action of IMPA2. Significantly, the inhibition of IMPA2 activated ERK phosphorylation, and its inhibitory effects can be restored by using selective ERK inhibitor, FR180204. In conclusion, IMPA2 acts as an oncogene in the proliferation and migration of cervical cancer. IMPA2 downregulated ERK phosphorylation to promote cervical cancer. These findings identify a new mechanism underlying cervical cancer and suggest a regulating effect of IMPA2 in MAPK signaling pathway.
\end{abstract}

\section{Introduction}

Cervical cancer is the fourth leading cause of cancerassociated death regarding gynecological malignancies worldwide ${ }^{1}$, even though prevention and treatment have rapidly developed recently ${ }^{2,3}$. As we all know, the most significant cause of cervical cancer is persistent infection of human papillomavirus (HPV). HPV is detected in $99 \%$ of cervical cancer patients ${ }^{4}$, but most women infected with HPV failed to develop invasive cervical cancer ${ }^{5}$. Therefore, comprehensive understanding of molecular

\footnotetext{
Correspondence: Min Wang (wangmin0000@csu.edu.cn)

'Department of Laboratory Medicine, Second Xiangya Hospital, Central South University, Changsha, Hunan, China

${ }^{2}$ Department of Obstetrics and Gynecology, Second Xiangya Hospital, Central

South University, Changsha, Hunan, China

These authors contributed equally: Kan Zhang, Lei Liu

Edited by J.-E. Ricci
}

mechanisms underlying cervical cancer will promote the earlier diagnosis and effective treatment.

Genetic mutations were proved to play important roles in development of cervical cancer ${ }^{6}$. RNA sequencing provides an efficient and comprehensive method to identify the key genes and molecular pathways involved in cervical cancer pathogenesis ${ }^{7,8}$. Cervical carcinoma in situ (CCIS), without stromal invasion, and superficially invasive carcinomatosis, is the earliest stage in cancer progression. By comparing gene expression profiles of CCIS and adjacent normal tissues, it is possible to look for the most direct evidence of tumorigenesis and help early diagnosis. Here, we sought potential oncogenes of cervical cancer, by comparing mRNA expression profiles of tissue samples of CCIS with adjacent normal cervical tissue. $I M P A 2$ gene was discovered to be significantly upregulated in CCIS tissues. IMPA2 located on chromosome 
Table 1 Clinical characteristic of cervical cancer patients enrolled in this study.

\begin{tabular}{|c|c|c|c|c|c|}
\hline ID & $\begin{array}{l}\text { Age at } \\
\text { diagnosis } \\
\text { (years) }\end{array}$ & $\begin{array}{l}\text { Pathological } \\
\text { type }\end{array}$ & $\begin{array}{l}\text { FIGO } \\
\text { stage }\end{array}$ & HPV types & $\begin{array}{l}\text { Reproductive } \\
\text { history } \\
\text { (pregnancy- } \\
\text { birth-abortion) }\end{array}$ \\
\hline 1 & 47 & $\mathrm{CIS}$ & 0 & $51+/ 52+/ 44+$ & $5-1-4$ \\
\hline 2 & 51 & $\mathrm{CIS}$ & 0 & $33+/ 53+$ & $5-2-3$ \\
\hline 3 & 43 & $\mathrm{CIS}$ & 0 & $16+/ 53+$ & $2-1-1$ \\
\hline
\end{tabular}

CIS carcinoma in situ, FIGO International Federation of Gynecology and Obstetrics, HPV Human Papilloma Virus.

18p11.2, encodes myo-inositol monophosphatase 2 (IMPA2) with 288 amino acids ${ }^{9}$. IMPA2 has intrinsic IMPase activity that is completely dependent on magnesium $^{10}$, and is involved in phosphatidylinositol signaling pathway, which is associated with cellular activities such as metabolism, secretion, cell growth, and differentiation $^{11}$. Therefore, we speculated that IMPA2 may be a cancer-promoting gene in cervical cancer. However, most studies about IMPA2 focused on neuropsychiatric diseases and the pharmacological action of Lithium ${ }^{10,12,13}$. Recently, French et al. ${ }^{14}$ found that IMPA2 expression might affect accumulation of methotrexate polyglutamates (MTXPGs) in leukemia. In addition, Lin et al $^{15}$ indicated that IMPA2 downregulation leads to poor outcomes in clear cell renal cell carcinoma (ccRCC). This is contrary to our speculation on the role of IMPA2 in cervical cancers. As there are few published articles about the role of IMPA2 gene in cervical cancer, this study performed both in vitro and in vivo studies to discuss the relationship between cervical cancer and IMPA2.

Here, we discussed the role of IMPA2 and found that IMPA 2 promoted the ability of proliferation, metastasis, and in vivo tumorigenesis of cervical cancer cells. Further proteomic analysis was performed to discuss the possible mechanisms regulated by IMPA2 in cervical cancer. The present study is proposed to identify the potential cancerpromoting action of IMPA2 in cervical cancer and explore possible pathways controlled by IMPA2 to further understanding the molecular mechanisms underlying cervical cancer.

\section{Material and methods}

\section{Tissue sample selection}

Cervical carcinoma in situ (CCIS) and adjacent normal tissues were obtained from three patients who underwent radical hysterectomy at the Second Xiangya Hospital, Central South University. All patients were diagnosed by multipoint biopsy. Table 1 showed the clinical characteristics of the patients. After tumor purity analysis performed by ESTIMATE algorithm, samples were collected for transcriptome analyses ${ }^{16}$. None of the three patients had received adjuvant therapy (chemotherapy or radiotherapy) prior to uterectomy. In addition, the other 58 patients with cervical cancer were enrolled from September 2015 to December 2017. Clinicopathologic features of the patients were shown in Table S1. Among them, 57.4\% (35/61) were positive for HPV16, 19.7\% (12/ 61) for HPV 58, 11.5\% (7/61) for HPV 33, 11.5\% (7/61) for HPV 52, 8.2\% (5/61) for HPV51, and 8.2\% (5/61) for HPV18. This study was approved by the Joint Ethics Committee of the Central South University Health Authority and performed following national guidelines. Written informed consent was obtained from all the patients. The clinical staging and clinicopathological classifications were determined according to the International Federation of Obstetrics and Gynecology (FIGO). Paired cervical cancer and adjacent normal tissues were collected at surgery, immediately frozen in liquid nitrogen and stored until total RNA or proteins were extracted.

\section{Immunohistochemistry staining analysis}

The immunohistochemical staining procedure was performed as previously described ${ }^{17}$. Cervical cancer samples for IMPA2 detecting were obtained from the Second Xiangya Hospital of Central South University. Samples for ERK and p-ERK detecting were xenografts from mice. The staining positivity was determined by the following formula: IRS = intensityscore $\times$ quantity score. The percentage of positive cells was divided into five score ranks: $<10 \%(0), 10-25 \%(1), 25-50 \%(2), 50-75 \%(3)$, and $>75 \%$ (4). The intensity of staining was divided into four score ranks: no staining (0), light brown(1), brown (2), and dark brown (3). Two different pathologists evaluated all the specimens in a blinded manner. The antibodies used were as follows: anti-IMPA2 rabbit monoclonal antibody (1:100, GeneCopoeis, USA); anti-ERK (1:500, Abcam, UK), and anti-pERK (1:400, Cell Signaling, USA).

\section{Cell culture}

Cervical cancer cell line SiHa (\#BNCC337881) and normal cervical epithelial cell line, HcerEpic (\#BNCC340373) were purchased from the Cell Bank of BeNa culture collection (Beijing, China). Cervical cancer cell line HeLa (\#GCC-UT0002CS) was purchased from the Cell Bank of Genechem (Shanghai, China). The cell line was cultured in Dulbecco's modified eagle medium (DMEM) (Gibco, Grand Island, NY, USA) supplemented with $10 \%$ fetal bovine serum (FBS) (Gibco, Grand Island, NY, USA) and $1 \%$ antibiotics at $37^{\circ} \mathrm{C}$ in an atmosphere containing $5 \% \mathrm{CO}_{2}$.

\section{IMPA2 silencing}

The short hairpin RNA (shRNA) targeting IMPA2 mRNA (shIMPA2) and the negative control were 
obtained from Ribobio (Guangzhou, China). Sequences of shIMPA2 were listed as follow: forward $5^{\text {"-CCGG }}$ GCCTTACAGACGATTAACTATCTCGAGATAGTTA ATCGTCTGAAGGCTTTTTG-3'; Reverse 5'-AATTC AAAAAGCCTTACAGACGATTAACTATCTCGAGAT AGTTAATCGTCTGTAAGGC-3'. The lentivirus was packaged using GV115 vector, pHelper 1.0 vector and pHelper 2.0 vector, as well as Lipofectamine 2000 (Invitrogen, Carlsbad, CA, USA; Thermo Fisher Scientific) for the HEK293T cell and then collected after $48 \mathrm{~h}$. The SiHa cell line was infected with lentivirus and polybrene (1:500; Shanghai Ji Kai Gene Chemical Technology Co., Ltd., Shanghai, China) according to the manufacturer's instructions. The expression change of IMPA2 was determined by reverse-transcription polymerase chain reaction (RT-PCR) and Western blotting at $72 \mathrm{~h}$ after transfection.

\section{In vivo tumor formation assay}

A total of 14 health female BALB/c (nu/nu) nudes (4 weeks, 20-23 g) were purchased from Shanghai Lingchang Biotechnology limited company and fed in SPF Animal Laboratory of Central South University with sterile water and food. The mice were divided into 2 groups based on the randomized table, Normal Control (shCtrl) group and IMPA2 RNAi (shIMPA2) group. In all, $5 \times 10^{6}$ indicated stable cell lines were subcutaneously injected into right flank of BALB/c (nu/nu) mice in each group. Tumor sizes and weights were measured once a week. Mice were examined by in vivo fluorescence imaging system (Lumina LT, Perkin Elmer, USA) and killed for the analysis of tumor burden after 4 weeks. The tumors were stripped for follow-up experiments. All the experiments were strictly accordant with the care and use guidelines of experimental animal and approved by the Animal Protection Committee.

\section{RNA isolation and quantitative real-time PCR}

Total RNA was extracted using the Trizol reagent (Sangon Biotech, Shanghai, China). RNA $(1 \mu \mathrm{g})$ was reverse transcribed into cDNA using Transcriptor First Strand cDNA Synthesis Kit (Roche Diagnostics, Germany) according to the supplier's instructions. Quantitative real-time PCR analysis was performed with Stratagene Mx3000P qPCR system (Agilent Technologies, USA) using Thunderbird qPCR Mix (TOYOBO, Japan). CDNA samples were tested in triplicate and glyceraldehyde-3-phosphate dehydrogenase (GADPH) was used as a reference gene. The expression of IMPA2 was quantified by measuring $\mathrm{Ct}$ values and normalized using the $2^{-\Delta \Delta C t}$ method relative to GAPDH. The primer pairs used for qRT-PCR were designed using the primer3 program. Primers used were as follows: IMPA2 forward, 5'-GAAACCTCTCTCGCAACTCAG-3' , reverse, 5' -GGG
CAGGACAGATCATCAGAA-3'. GADPH forward: 5'GAACGGGAAGCT CACTGG-3' ${ }^{\prime}$, reverse, 5-GCCTGC TTCACCACCT TCT-3'.

\section{Western blotting analysis}

Details of Western blotting were previously described ${ }^{18}$. Cells at $80-90 \%$ confluence were lysed on ice in radioimmunoprecipitation assay buffer (RIPA; keygen biotech, China) containing PMFS complete protease inhibitor cocktail (keygen biotech, China). Protein concentration was determined by the BCA assay (keygen biotech, China). Equal protein samples $(10 \mu \mathrm{g})$ were separated on $12 \%$ sodium dodecyl sulfate (SDS)/polyacrylamide gels, and transferred onto $0.45 \mu \mathrm{m}$ polyvinylidene difluoride (PVDF) membranes (Immobilon-P; Millipore, Bedford, MA, USA). The antibodies used were as follow: antiIMPA2 rabbit monoclonal antibody (1:1000; GeneCopoeis, USA); anti-ERK (1:10,000, Abcam, UK), anti-p38 $\alpha$ (1:500, BBI China), anti-JNK1/2/3 (1:500, BBI China), p-ERK (1:500, Cell Signaling, USA), p-p38 $\alpha$ (p-Thr180/ Tyr182, 1:500, BBI China), and p-JNK1/2/3 (p-Th183/ Ty185, 1:500, BBI China). Horseradish peroxidase (HRP)conjugated goat anti-rabbit immunoglobulin G (1:1000; BBI China) was used as second antibody and anti-GAPDH mouse monoclonal antibody (1:5000; BBI China) as a loading control. The final protein expression was detected by enhanced chemiluminescence (Bio-rad, Berkeley, CA, USA) according to the manufacturer's suggested protocols. The band quantification was conducted using ImageJ (National Institutes of Health, Bethesda, MA, USA).

\section{CCK-8 cell viability assays}

Cells were seeded into a 96-well plate at $2 \times 10^{3}$ cells per well with $100 \mu \mathrm{l}$ cultured medium and cultured for 24,48 , 72 , and $96 \mathrm{~h}$ at $37^{\circ} \mathrm{C}, 5 \% \mathrm{CO}_{2}$. The cell viability was determined with CCK8 assay as previously described ${ }^{19}$. Each process was repeated three times.

\section{Colony formation assay}

Cells (1000/well) were plated in 6-well plates and cultured for 2 weeks. The colonies were washed with PBS three times and fixed with $4 \%$ formaldehyde for $10 \mathrm{~min}$. Then, the colonies were stained with $1 \%$ crystal violet for $10 \mathrm{~min}$. After washing, the colonies were counted. The experiment was carried out in triplicate for each cell line.

\section{Wound healing and transwell migration assays}

In the wound healing assay, cells $\left(2 \times 10^{6} /\right.$ well $)$ were seeded in 6 -well plates. When the cells were $90 \%$ confluent, they were serum-starved for $24 \mathrm{~h}$. A linear wound was created in the confluent monolayer using a $10-\mu \mathrm{l}$ pipette tip. The wounds were observed and photographed immediately (time 0 ) and thereafter at 48 
(magnification, $\times 200$ ). Details of transwell migration assay was described previously ${ }^{20}, 2 \times 10^{4}$ cells in $200 \mu \mathrm{l}$ of serum-free medium were added to the top chamber of the transwell $(8-\mu \mathrm{m}$ pore size, BD Biosciences, New Jersey, USA). The bottom well contained growth medium with $20 \%$ FBS. After $24 \mathrm{~h}$ incubation at $37^{\circ} \mathrm{C}$, cells that had migrated to the lower face of the filters were fixed with $4 \%$ paraformaldehyde and stained with hematoxylin and finally counted under a magnification of $\times 200$ (10 random fields/well). Each experiment was repeated at least 3 times.

\section{Sample preparation for proteomic measurement}

The cultured cells were scraped and collected in $1.5 \mathrm{~mL}$ Eppendorf tubes. Then, samples were sonicated three times on ice using a high intensity ultrasonic processor (Scientz) in lysis buffer (8 M urea, 1\% Protease Inhibitor Cocktail). The remaining debris was removed by centrifugation at $12,000 \times g$ at $4{ }^{\circ} \mathrm{C}$ for $10 \mathrm{~min}$. Finally, the supernatant was collected and the protein concentration was determined with BCA kit. The protein solution was reduced with $5 \mathrm{mM}$ dithiothreitol for $30 \mathrm{~min}$ at $56^{\circ} \mathrm{C}$ and alkylated with $11 \mathrm{mM}$ iodoacetamide for $15 \mathrm{~min}$ at room temperature in darkness. The protein sample was then diluted by adding $100 \mathrm{mM}$ NH4HCO3 to urea concentration <2 M. Finally, trypsin was added at 1:50 trypsin-to-protein mass ratio for the first digestion overnight and 1:100 trypsin-to-protein mass ratio for a second 4-h-digestion.

\section{LC-MS/MS analysis}

The tryptic peptides were separated by an EASY-nLC 1000 UPLC system and then subjected to NSI source followed by tandem mass spectrometry (MS/MS) in Q Exactive TM Plus (Thermo) coupled online to the UPLC. The resulting MS/MS data were processed using Maxquant search engine (v.1.5.2.8). Tandem mass spectra were searched against SwissProt Human database concatenated with reverse decoy database. Trypsin/P was specified as cleavage enzyme allowing up to two missing cleavages. The mass tolerance for precursor ions was set as $20 \mathrm{ppm}$ in First search and $5 \mathrm{ppm}$ in Main search, and the mass tolerance for fragment ions was set as $0.02 \mathrm{Da}$. Carbamidomethyl on Cys was specified as fixed modification, and oxidation on Met was specified as variable modifications. False positive rate (FDR) was adjusted to $<1 \%$ and minimum score for peptides was set $>40$.

\section{Bioinformatics analysis of differentially expressed proteins}

Firstly, protein ID of the differentially expressed proteins (DEPs) were converted to UniProt ID according to the UniProt-GOA database (www. http://www.ebi.ac.uk/ GOA/) and then mapped to Gene Ontology (GO) IDs. Proteins were classified by GO annotation based on three categories: biological process, cellular component, and molecular function. Then, Kyoto Encyclopedia of Genes and Genomes (KEGG) database was used to annotate protein pathway and to identify enriched pathways. KEGG online service tools KAAS and KEGG mapper help annotate protein's KEGG database description and map the annotation result on the KEGG pathway database. Finally, a two-tailed Fisher's exact test were used to test the enrichment of the DEP against all identified proteins. A corrected $p$-value $<0.05$ was considered significant.

\section{Statistical analysis}

The results were analyzed using SPSS 22.0 (Chicago, IL, USA) and GraphPad Prism 6 software (GraphPad Software, San Diego, CA, USA). The data were expressed as the mean \pm SD. The $t$-test and one-way ANOVA Tukey's posthoc test were used to analyze the statistical significance of parametric data from two and more independent samples, respectively. Non-parametric Wilcoxon Signed-Ranks test, Friedman test, Mann-Whitney $U$ test and Kruskal-Wallis test were used to analyze data from two related samples, three or more related samples, two independent samples and three or more independent samples, respectively.

\section{Results}

\section{IMPA2 is overexpressed in cervical cancer}

Comparing the gene expression profiles of CCIS and adjacent normal cervical samples resulted in 1555 differentially regulated genes at $P<0.05$. Among the significant genes, 961 genes were up-regulated and 594 were downregulated in CCIS (Fig. 1a). Of these, IMPA2 gene was upregulated 11 times. Further real-time PCR was used to determine the IMPA2 expression pattern in 61 pairs of cervical cancer tissues and their matched adjacent nontumor cervical tissues. IMPA2 is significantly overexpressed in cervical cancer samples $(P<0.0001$, Fig. $1 b)$. Overexpression of more than 2-fold was displayed in 47.5\% (29 of 61) of the cervical cancer samples compared with non-tumor samples (Fig. 1d). Analysis of a public CESC (Cervical squamous cell carcinoma) dataset from The Cancer Genome Atlas (TCGA) (https://tcga-data.nci. nih.gov/tcga/tcgaDownload.jsp) also showed significant IMPA2 overexpression (Fig. 1c) ${ }^{21}$. Expression of IMPA2 was also compared among cervical cancer cell lines and normal cervical epithelial cell line to prove the overexpression (Fig. 1e, f). Moreover, increased protein level of IMPA2 was also observed in paired cervical cancer samples by immunohistochemistry (IHC; Fig. 1g).

\section{Inhibition of IMPA2 suppresses tumorigenesis of SiHa cells in vivo}

SiHa cells infected with IMPA2 shRNA or shRNA control were subcutaneously injected into each flank of nude mice. The tumor size of each nude mouse was 
A

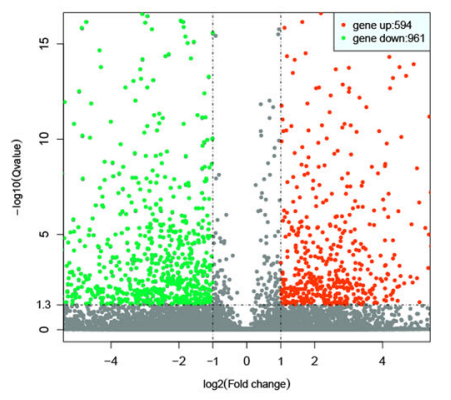

D

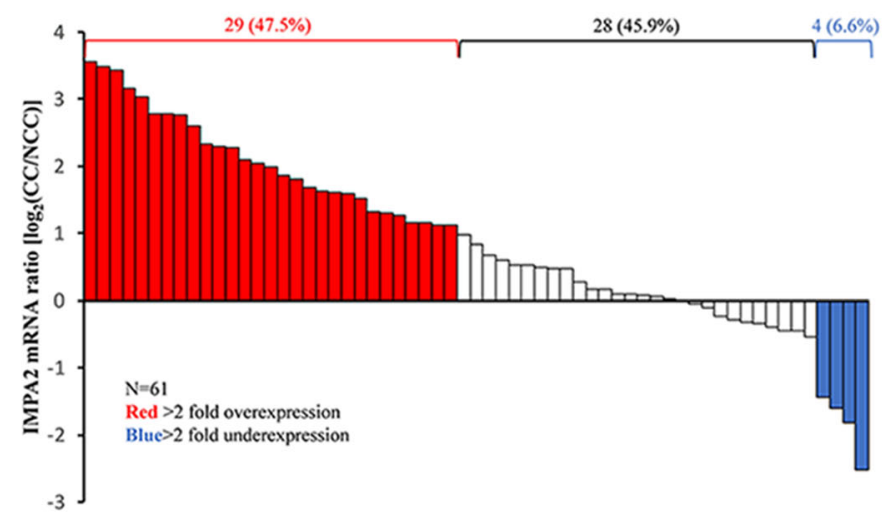

G

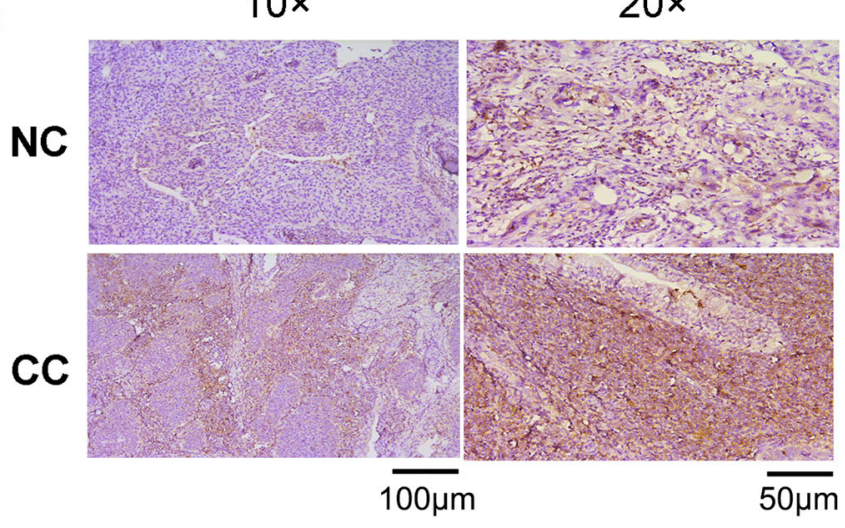

B

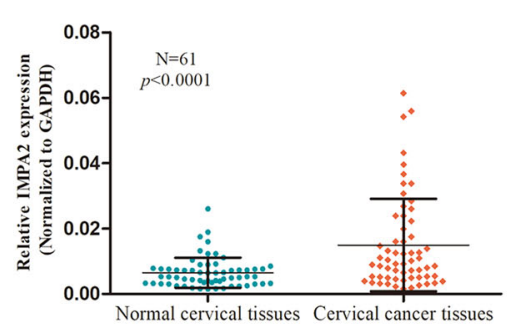

C

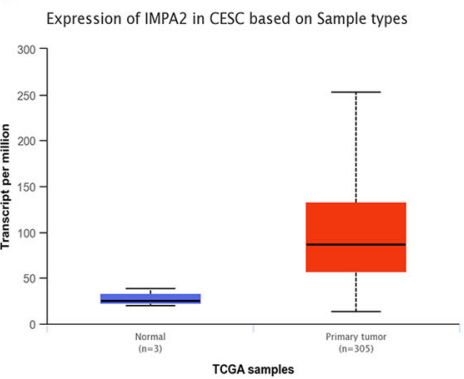

E

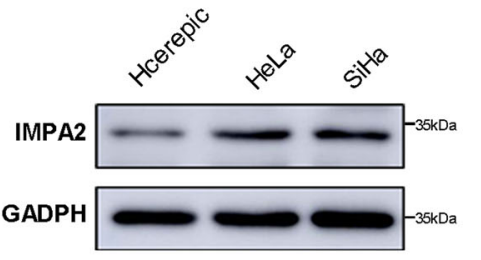

F
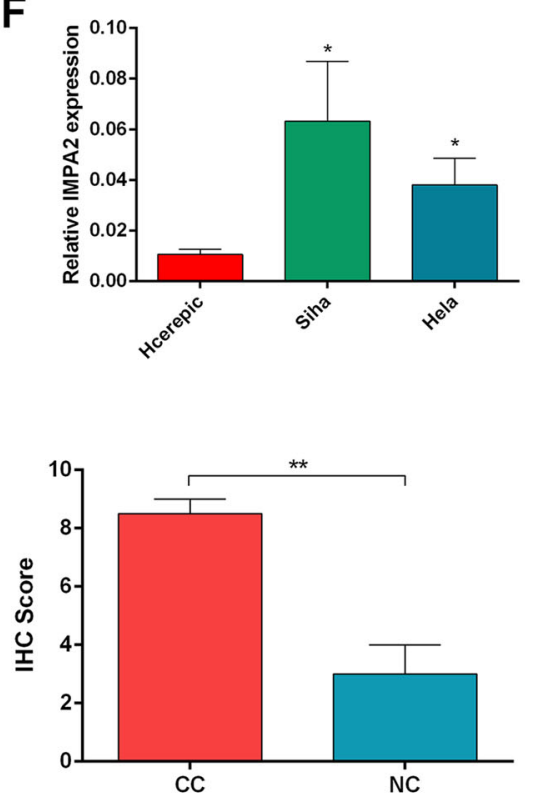

Fig. 1 IMPA2 was overexpressed in cervical cancer tissue and cell lines. a Volcano plot of differentially expressed genes. b Relative expression of IMPA2 in cervical cancer tissues $(n=61)$ compared with that of adjacent normal tissues $(n=61)(P<0.0001)$. c Expression of IMPA2 in normal and CESC tissues using public data from the TCGA-CESC dataset (Normal vs Primary tumor: $P=0.0004$ ). $\mathbf{d}$ Waterfall plot analyses of IMPA2 mRNA levels in CC and matched non-tumor cervical specimens. Red and blue bars represent samples that show a relative IMPA2 fold change of $\geq 2$ overexpression and underexpression, respectively (CC/NT). e IMPA2 expression was determined using western blotting and qRT-PCR (f) in SiHa and Hela cells. g Immunohistochemical (IHC) detection of IMPA2 in normal cervical samples (NC) and cervical cancer samples and the Immunoreactivity scores of IMPA2 were shown. Scale bar, 100 or $50 \mu \mathrm{m}$. Values were all presented as mean values \pm SD. ${ }^{*} P<0.05$.

measured and record weekly to draw the growth curve (Fig. 2b). After 5 weeks, all the mice were anesthetized to perform whole-body fluorescent imaging. As shown in Fig. 2a, no obvious metastasis was discovered in both groups. After that, all the mice were killed to harvest the xenografts. The tumor size and weight were measured and compared between groups. The IMPA2 silencing group showed significantly smaller tumor size $(P=0.0274)$ and lighter average weight $(P=0.0123)$ than the control group (Fig. 2c, d). 
A

Normal control

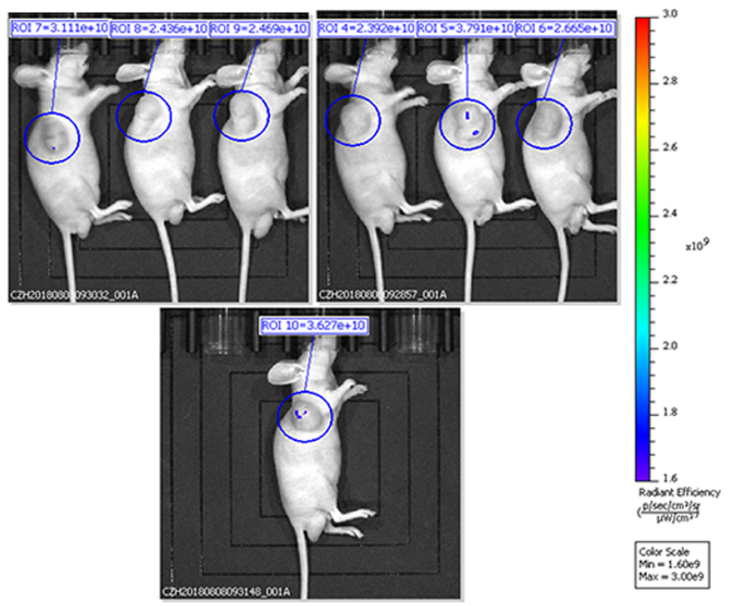

B

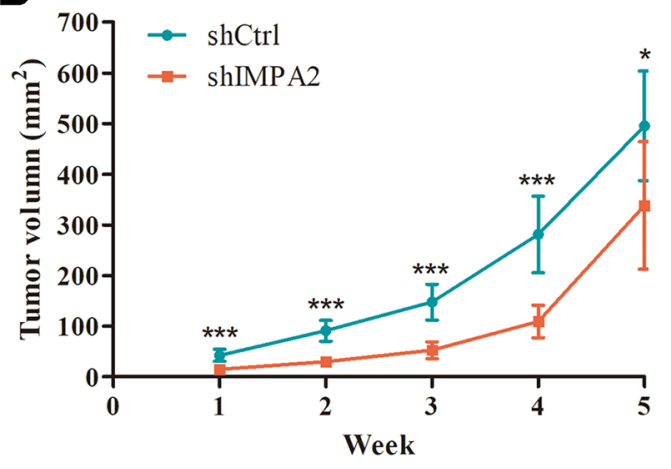

IMPA2 silencing

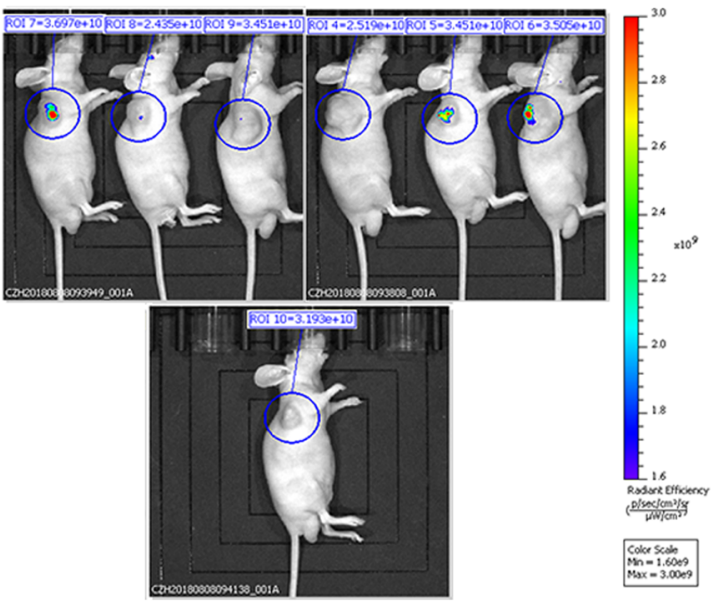

C

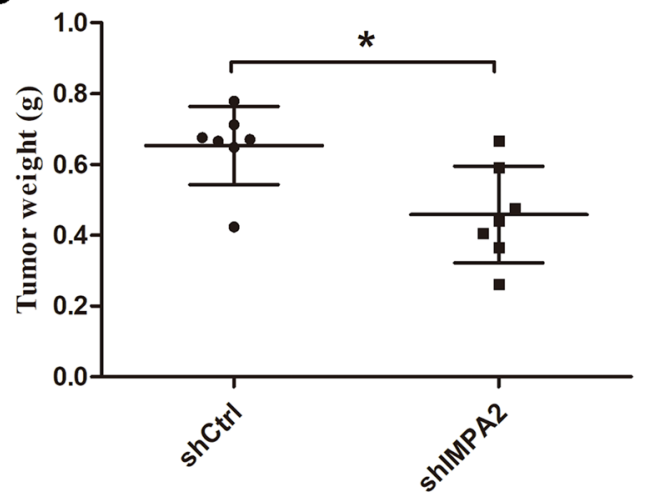

D

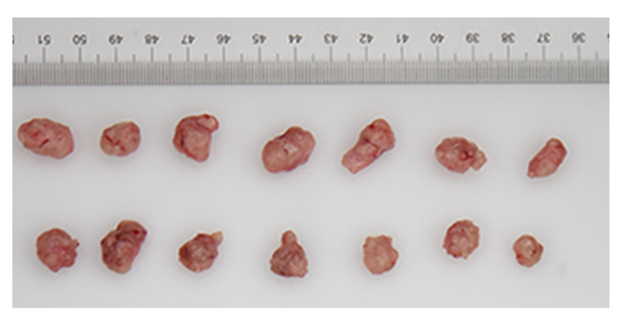

Normal control

IMPA2 silencing

Fig. 2 Inhibition of IMPA2 suppresses tumor growth in vivo. a Nude mice were transplanted subcutaneously with SiHa cells transfected with IMPA2 shRNA or the control shRNA. After 35 days, the mice were killed. Whole-body fluorescent imaging was performed for each nude mouse, and the representative pictures was recorded and presented. b Tumor volumes were measured weekly from week one to five post-injection. c Tumor weights were measured after the mice were killed. Bars indicate mean values \pm SD. $\mathbf{d}$ A representative picture of the morphology of tumor xenografts after excision at the 35th day. ${ }^{*} P<0.05$.

IMPA2 promotes the proliferation and clonogenicity of cervical cancer cells in vitro

The role of IMPA2 in cervical cancer cells were detected after transfected with overexpressed plasmids and small interfering RNA (siRNA) lentivirus. The downregulation and overexpression efficiency were verified using qRT-
PCR and western blotting (Fig. 3a, b). CCK8 cell viability assay (Fig. 3c, d) and colony formation assay (Fig. 3e) were performed to evaluate cell growth. As a result, silencing IMPA2 in SiHa and HeLa cells both significantly slowed the cell growth. Overexpression of IMPA2 failed to enhance the cell growth in both cervical cancer cells. 
A

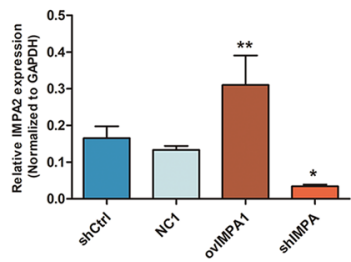

C

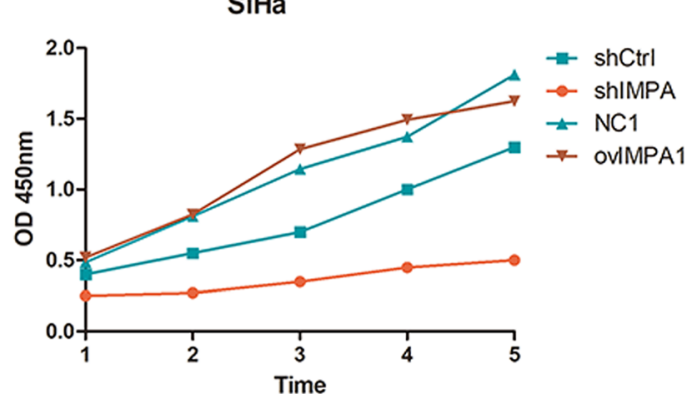

E

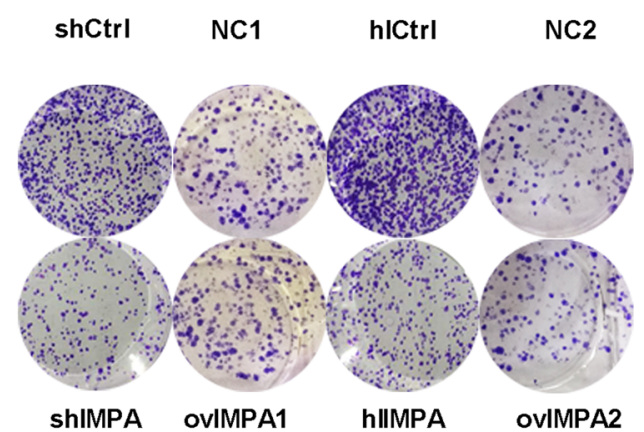

B

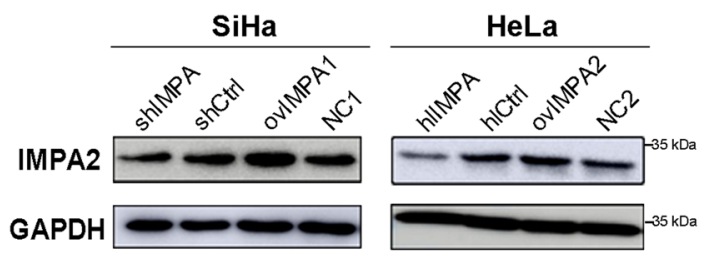

D
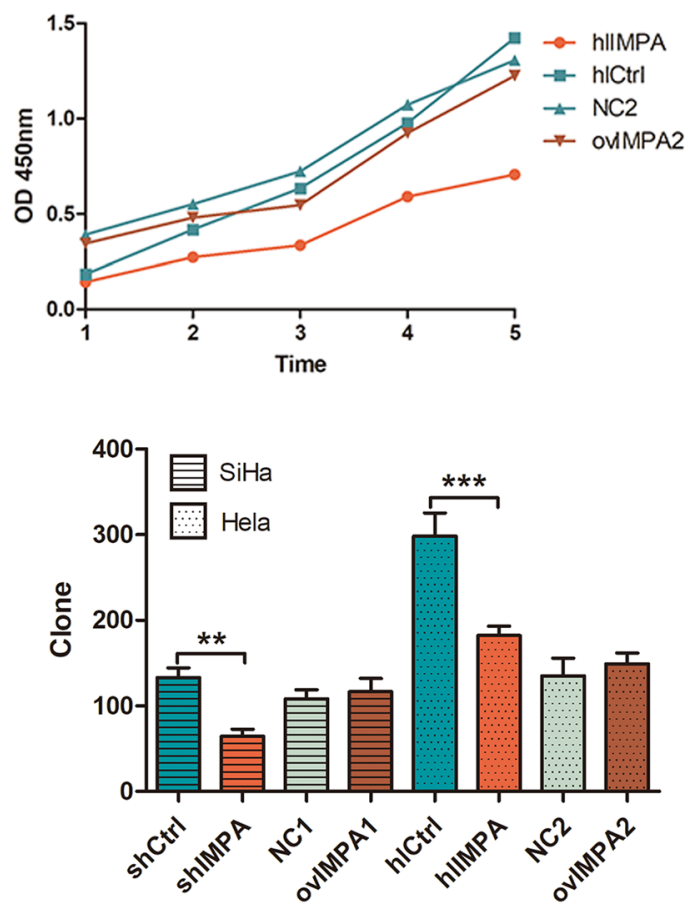

Fig. 3 IMPA2 downregulation inhibits proliferation and clonogenicity of SiHa and Hela cells in vitro. a, b ShRNA silence of IMPA2 dramatically downregulated IMPA2 expression at the RNA level and protein level when compared to the negative control shRNA (shCtrl) in SiHa cell line by qRTPCR and western blotting. $\mathbf{c}$, d CCK-8 assay was performed to measure proliferation in IMPA2 silencing and overexpressing SiHa or Hela cells and in the respective control cells. e The cell clonogenicity of IMPA2 silencing and overexpressing SiHa or HeLa cells and the respective control cells was analyzed using Celigo Image Cytometry. The data represent the mean \pm SDs of three replicates, ${ }^{* * *} P<0.001$.

Similarly, fewer and smaller colonies appeared in cells treated with IMPA2-siRNA than those in controls $(P<$ 0.01 ). In contrast, no significant difference was found between the IMPA2 overexpression group and the control group.

\section{IMPA2 promotes the migration of cervical cancer cells in vitro}

Wound-healing assay and transwell migration assay were conducted to examine the effect of IMPA2 on the migration and invasion of cervical cancer cells. IMPA2 silencing slowed the speed with which both cervical cancer cells filled the scratch, in comparison to the control in an obvious manner (Fig. 4b). The transwell assays yielded the similar results $(P<0.001$, Fig. 4 a). On the contrary, the cell migration capacity was improved (Fig. 4b) and the number of invaded cells were significantly higher $(P<0.001$, Fig. 4 a $)$ in IMPA2 overexpression group.

\section{Pathway analysis of differentially expressed protein between shCtrl and shIMPA2}

The proteomic profile of shCtrl and shIMPA2 (Fig. 5) was determined by LC-MS/MS. A total of 4364 proteins were identified. Using a cutoff value 1\% FDR for statistical significance, 267 proteins were found to be differentially expressed: 112 significantly overexpressed (shIMPA2/shCtrl ratio 21.50 ) and 155 underexpressed 


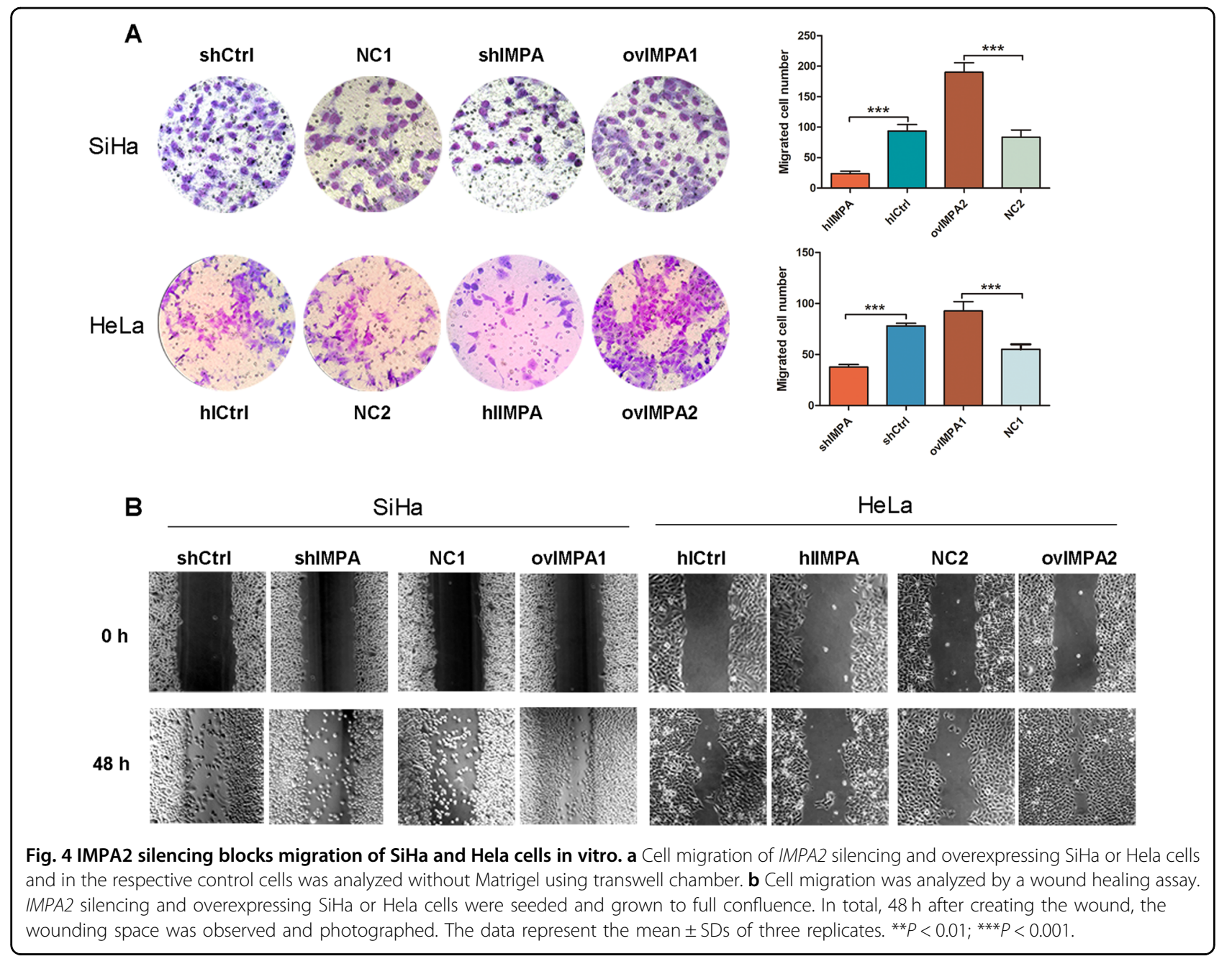

(shIMPA2/shCtrl ratio $\leq 0.50$ ). Further bioinformatics analysis was then performed to assess whether the DEPs were related to specific molecular pathways. In the Gene Ontology (GO) enrichment, 14 biological processes, 8 molecular functions, and 8 cellular components were enriched with statistical significance (Fig. 5a-c). Main biological processes include DNA repair, glycoprotein biosynthesis and metabolism, RNA biosynthesis, and cell death process. The analysis by cellular components showed that IMPA2 silencing produced a more profound impact on autophagosome-related structure and secretory granule membrane. Moreover, the analysis of molecular functions showed that proteins involved in the binding of transcript, protein kinase and drug, and folic acid, as well as many enzymatic activities like oxidoreductase and DNA polymerase. Cluster analysis based on shIMPA2/shCtrl ratio showed the involved biological processed in more detail (Fig. 5b). KEGG pathway enrichment analysis indicated that most of the DEPs were related to Fanconi anemia pathway, one carbon pool by folate, viral myocarditis, legionellosis, and MAPK signaling pathway (Fig. 5d). Eight DEPs involved in the MAPK pathway were significantly changed after inhibition of IMPA2, including four upregulated proteins (HSPA1L, RAP1A, NFKB1 and TAB1) and four downregulated proteins (MAP2K3, EPHA2, IL1RAP, and ECSIT). The fold change and primary information of these proteins were detailed in Table 2.

\section{Inhibition of IMPA2 activates the ERK/MAPK pathway}

To understand further the mechanisms responsible for IMPA2-related cancer progression, MAPK signaling pathways were analyzed by Western blotting based on the proteomic profile results. We found that compared with the shCtrl group, IMPA2 silencing significantly increased ERK phosphorylation in $\mathrm{SiHa}$ and Hela cells. Total levels ERK showed no statistical differences. And IMPA2 had little effect on JNK and p38 phosphorylation (Fig. 6a). Furthermore, silenced IMPA2 activating ERK expression was also been verified in inxenografts by Immunohistochemical staining (Fig. 6b). Thereafter, ERK inhibitor 


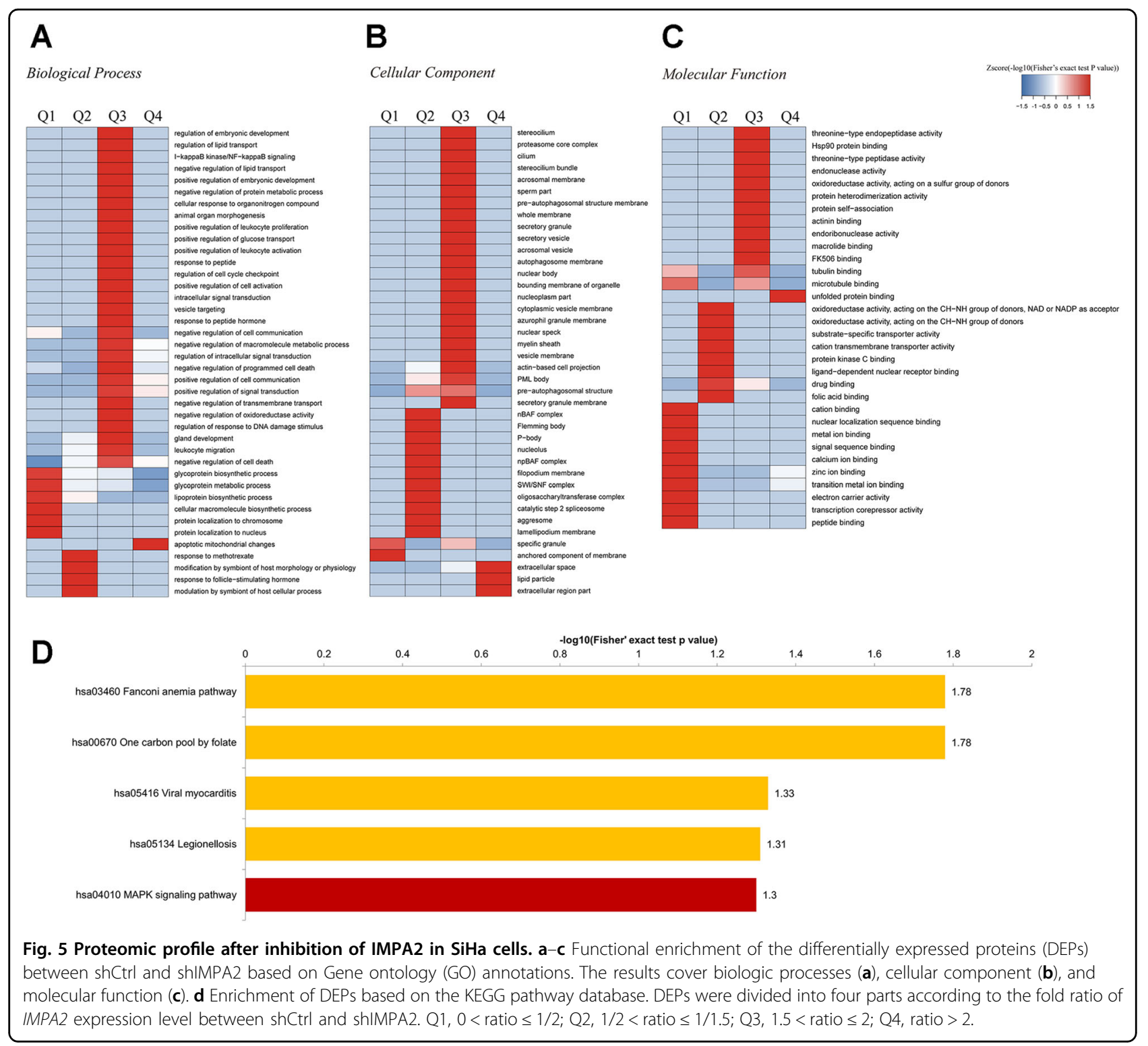

FR180204 $(10 \mu \mathrm{mol})$ was added in IMPA2-silenced SiHa and Hela cells for $48 \mathrm{~h}$. We found that FR180204 treatment significantly reversed the IMPA2-induced decrease in cell viability (Fig. 6d), cell migration and invasion in Hela cell (Fig. 6c, e), suggesting that activation of the ERK signaling pathway may result in inhibitory of proliferation and migration induced by IMPA2 in cervical cancer.

\section{Discussion}

Cervical cancer progression is a continuous process from normal cervical to cervical intraepithelial neoplasia (CIN) and finally to cancer. Various molecules were involved $^{22-25}$ besides the key role of human papillomavirus (HPV) infection. IMPA2 gene is a protein-coding gene for myo-inositol monophosphatase $2^{9}$. This monophosphatase is one of the key enzymes in inositol phosphate metabolism and finally convert monophosphate into free inositol through dephosphorylation. IMPA2 is received in many studies to be associated with neuropsychiatric diseases, like schizophrenia ${ }^{26}$, epilepsy ${ }^{27}$, bipolar disorder ${ }^{11,28,29}$, and Huntington's disease ${ }^{12,30}$. Also, IMPA2 inhibition is accepted as the therapeutic mechanism of lithium in bipolar disorders ${ }^{31}$. However, there is only one published article clearly demonstrated the relationship between IMPA2 expression and cancer progression. Lin et al. recently showed that IMPA2 downregulation constitutes a novel signature for cancer metastasis and poor outcomes in $\mathrm{ccRCC}^{15}$. On the contrary, our data of RNA sequencing (RNA-seq) showed an obvious upregulation of IMPA2 in cervical cancer. 


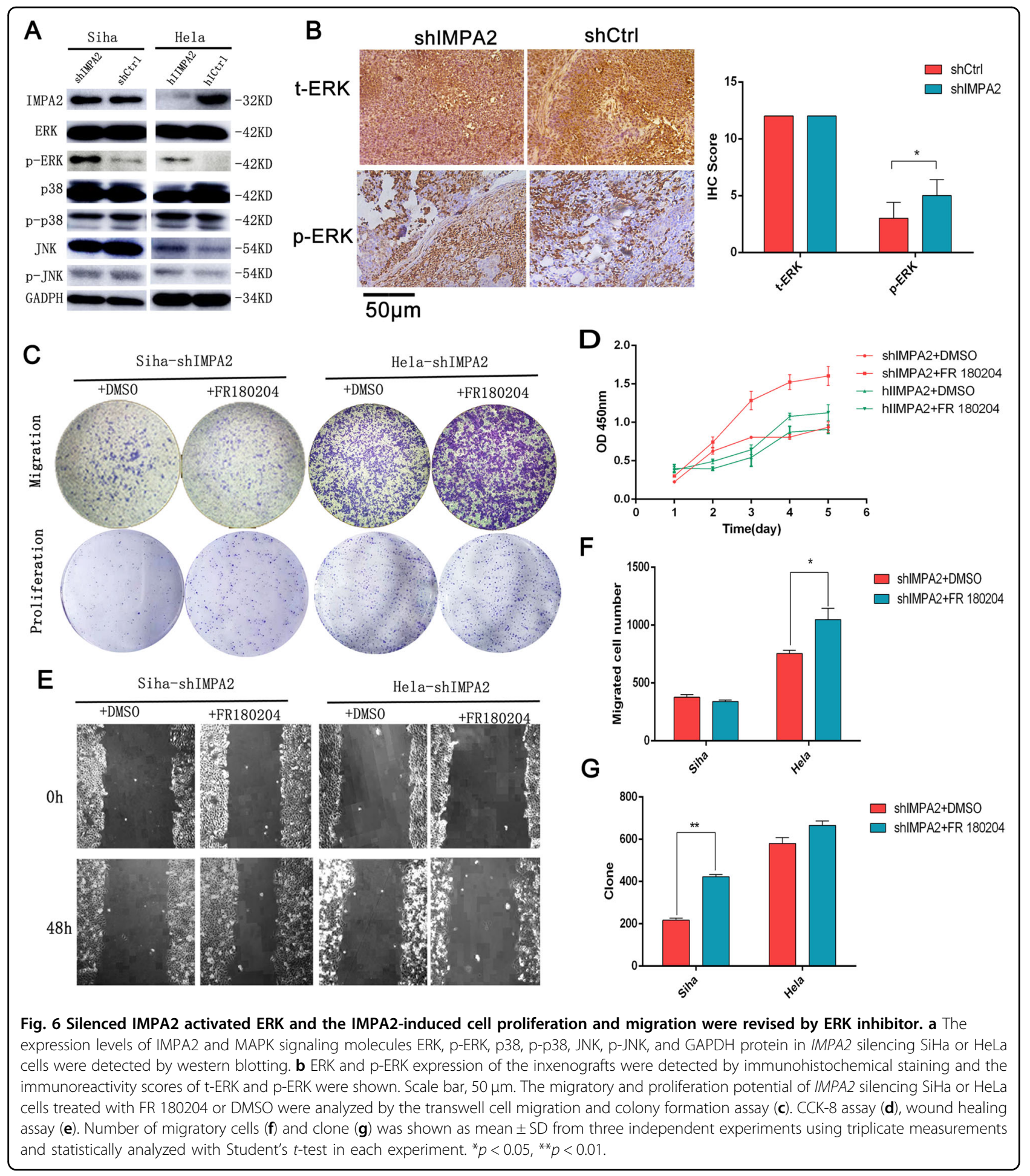

Therefore, our study investigated its ability to act as an oncogene and the possibility to be a potential diagnostic and prognostic biomarker as well as a therapeutic target in cervical cancer. In the present study, IMPA2 was upregulated in cervical cancer tissues when compared with pair matched normal tissues, which is consistent with the dataset of IMPA2 from TCGA. And about $47.5 \%$ $(29 / 61)$ overexpressed more than 2-fold. However, the upregulation of IMPA2 failed to correlate with clinical characteristics, HPV types and overall survival.

Our results demonstrated that inhibition of IMPA2 could slower the proliferation and promote the migration 
Table 2 Primary information and fold change of 8 DEPs involved in the MAPK pathway.

\begin{tabular}{llll}
\hline Gene name & Protein accession & Protein description & SI/SN ratio \\
\hline HSPA1L & P34931 & Heat shock 70-kDa protein 1-like & 2.016 \\
TAB1 & Q15750 & TGF-beta-activated kinase 1 and MAP3K7-binding protein 1 & 1.823 \\
RAP1A & P62834 & Ras-related protein Rap-1A & 1.573 \\
NFKB1 & P19838 & Nuclear factor NF-kappa-B p105 subunit & 1.551 \\
EPHA2 & P29317 & Ephrin type-A receptor 2 & 0.644 \\
MAP2K3 & P46734 & Dual specificity mitogen-activated protein kinase kinase 3 & 0.64 \\
IL1RAP & Q9NPH3 & Interleukin-1 receptor accessory protein & 0.587 \\
ECSIT & Q9BQ95 & "Evolutionarily conserved signaling intermediate in Toll pathway, mitochondrial" & 0.511 \\
\hline
\end{tabular}

SI scilenced IMPA2 group, SN negative control group.

of SiHa and Hela cells (Figs. 3, 4). And this effect of promoting tumorigenesis was also confirmed in vivo (Fig. 2). However, the in vivo result showed no migration or invasion in our shCtrl or shIMPA2 group. This can partly explain why IMPA2 was not associated with clinical and pathological characteristics well (Table S1). And as introduced previously, IMPA2 was identified upregulated in carcinoma in situ of cervix. We suspect that IMPA2 can be a promoting element in the early stage of cervical cancer, but the further migration or invasion still need other contributing factors. Collectively, the present study is the first report to demonstrate that IMPA2 may act as an oncogene in cervical cancer.

As the mechanism of promoting effect of IMPA2 has not been studied yet, we first traced back to the researches about mechanisms behind the role of IMPA2 in neuropsychiatric diseases. Most of the studies agreed that inhibition of IMPA2 might induce IP3 accumulation and /or inositol depletion, which can finally regulate autophagy to help treat neurodegenerative diseases or bipolar disorders ${ }^{12,30,31}$. However, the mechanism of IMPA2 in cancer is utterly unknown. We therefore performed proteomics analysis to look for clues of molecular regulation by IMPA2 in cervical cancer. In all, 267 proteins with different expression were detected, 149 of which have previously reported to be potentially related to biological processes of DNA damage repair, cell death, glycoprotein biosynthesis and metabolism, RNA biosynthesis, and embryonic development. Clustering analysis showed the involvement of cell death in more detail, as regulation of cell death, programmed cell death, and apoptotic mitochondrial changes were all significantly clustered.

The KEGG enrichment analysis for the identification of pathways unveils possible involvement of MAPK signaling pathway. MAPK plays an important role in regulating cell proliferation $^{32}$, differentiation ${ }^{33,34}$, cell cycle arrest $^{35}$, apoptosis $^{24,36,37}$, immune function ${ }^{38}$, and autophagy ${ }^{36,39}$. The MAPK signaling pathway is shared by four distinct cascades $^{40}$, including Jun amino-terminal kinases (JNK1/ 2/3), extracellular signal-related kinases (ERK1/2), p38MAPK and ERK5. The expression of above key molecules of $\mathrm{SiHa}$ and Hela cells pre and post inhibition of IMPA2 were compared by western blotting. Phosphorylation of ERK was activated after IMPA2 silencing. JNK $1 / 2 / 3$ and p38 remained unchanged (Fig. 6), suggesting that MAPK/ ERK signaling pathway take part in IMPA2-induced tumorigenesis of cervical cancer.

Many researches about chemotherapeutic drugs ${ }^{41,42}$, tumor-related gene ${ }^{43}$, microRNA $^{38}$, and LncRNA ${ }^{44}$ have shown multiple effects on cervical cancer by regulation of MAPK signaling pathway. However, most of the studies agreed that phosphorylation of ERK ${ }^{38,45}$ was upregulated to promote the proliferation or (and) suppress the apoptosis $^{33,46}$ of cervical cancer, while evidences showed contrary effect in this study (Fig. 6). When added ERK inhibitor in IMPA2-silenced cells, we could find that the proliferation capacity of Hela and migration of $\mathrm{SiHa}$ could not be revised completely (Fig. 6f, g). Therefore, we thought ERK cannot regulate tumorigenesis directly. Previous studies demonstrated that activation of ERK MAPK could also induce cytotoxicity or tumor inhibition in some cancers ${ }^{19,41,47}$. Some studies revealed that activation of ERK could promote apoptosis progress ${ }^{48,49}$. And as we all know, apoptosis is a common process of programmed cell death and plays a key role in maintaining cellular homeostasis in normal and cancer cells ${ }^{35,50}$. Therefore, we suspect that upregulated ERK decreased cervical cancer progression by activating apoptosis in IMPA 2 silencing cells. And the primary apoptosis assay by using flow cytometry had initially verified our hypothesis (Fig. 7), but this hypothesis needs to be confirmed in a further study.

In conclusion, IMPA2 is a novel tumor promotor that regulates ERK MAPK in cervical cancer. Thus, silencing IMPA2 could step down proliferation and metastasis of cervical cancer cells. It could also induce activation of 


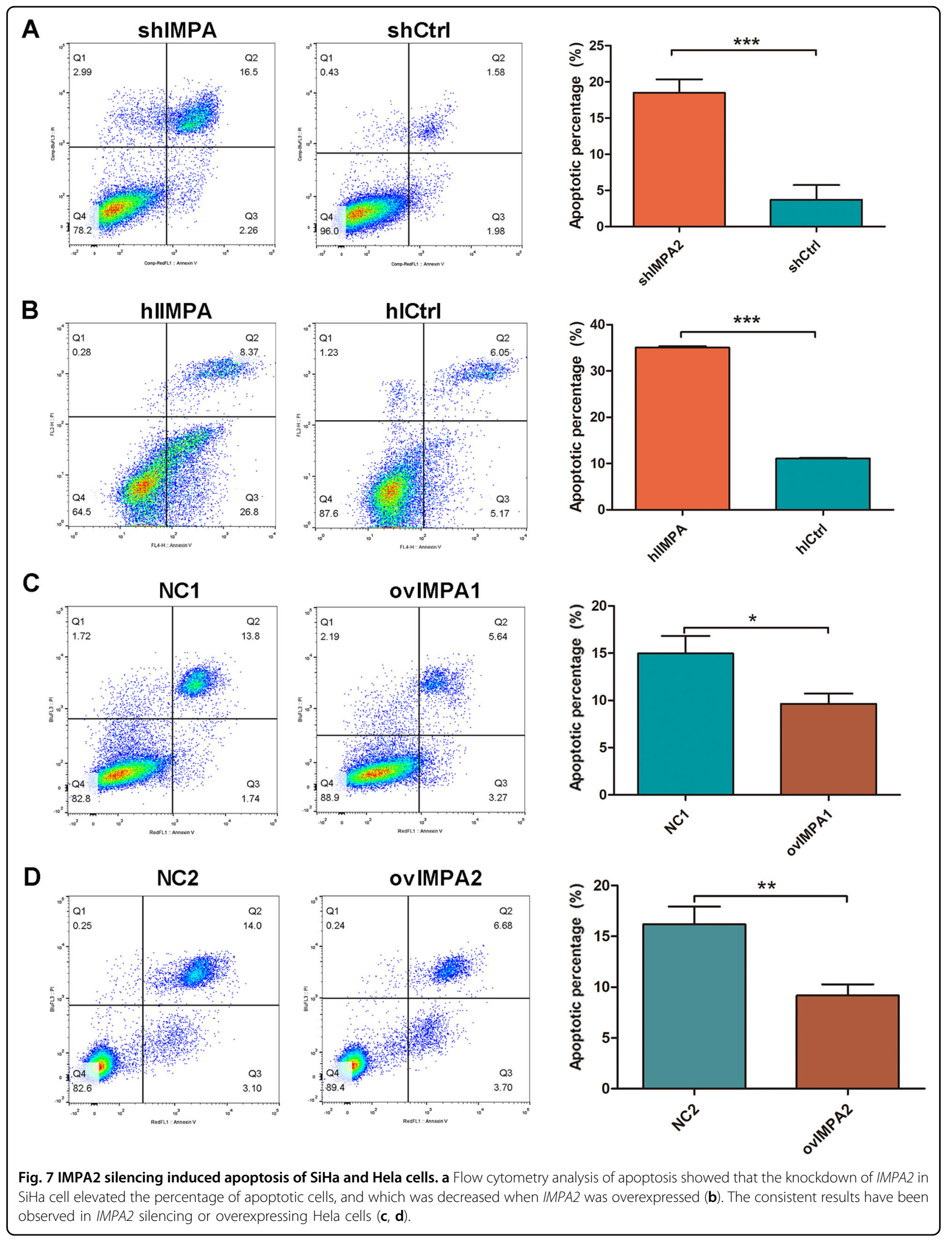


ERK. These findings provided promising insights into developing novel cancer therapies by inhibiting the IMPA2 in cervical cancer.

\section{Acknowledgements}

This work was supported by grants from National Natural Science Foundation of China (81470133), Natural Science Foundation of Hunan Province (2019JJ40426), Fundamental Research Funds for the central Universities of Central South University (2018zzts928), and Hunan Provincial Department of Finance (201895).

\section{Conflict of interest}

The authors declare that they have no conflict of interest.

\section{Publisher's note}

Springer Nature remains neutral with regard to jurisdictional claims in published maps and institutional affiliations.

Supplementary Information accompanies this paper at (https://doi.org/ 10.1038/s41419-020-2507-z).

Received: 11 February 2020 Revised: 10 April 2020 Accepted: 14 April 2020 Published online: 14 May 2020

\section{References}

1. Bray, F. et al. Global cancer statistics 2018: GLOBOCAN estimates of incidence and mortality worldwide for 36 cancers in 185 countries. CA Cancer J. Clin. 68, 394-424 (2018).

2. Petrosky, E. et al. Use of 9-valent human papillomavirus (HPV) vaccine: updated HPV vaccination recommendations of the advisory committee on immunization practices. MMWR Morb. Mortal. Wkly Rep. 64, 300-304 (2015).

3. Duenas-Gonzalez, A. \& Campbell, S. Global strategies for the treatment of early-stage and advanced cervical cancer. Curr. Opin. Obstet. Gynecol. 28, $11-17$ (2016)

4. Marth, C. et al. Cervical cancer: ESMO Clinical Practice Guidelines for diagnosis, treatment and follow-up. Ann. Oncol. 28, iv72-iv83 (2017).

5. Ho, G. Y., Bierman, R., Beardsley, L., Chang, C. J. \& Burk, R. D. Natural history of cervicovaginal papillomavirus infection in young women. N. Engl. J. Med. $\mathbf{3 3 8}$ 423-428 (1998).

6. $\mathrm{Wu}, \mathrm{Y}$. et al. Whole-exome and RNA sequencing reveals novel insights into the pathogenesis of HPV associated cervical cancer. Cancer Biomark https:// doi.org/10.3233/CBM-190055 (2019).

7. Yang, R. et al. Combined transcriptome and proteome analysis of immortalized human keratinocytes expressing Human Papillomavirus 16 (HPV16) oncogenes reveals novel key factors and networks in HPV-induced carcinogenesis. mSphere https://doi.org/10.1128/mSphere.00129-19 (2019).

8. Ojesina, A. I. et al. Landscape of genomic alterations in cervical carcinomas. Nature 506, 371-375 (2014).

9. Yoshikawa, T., Turner, G., Esterling, L. E., Sanders, A. R. \& Detera-Wadleigh, S. D. A novel human myo-inositol monophosphatase gene, IMP.18p, maps to a susceptibility region for bipolar disorder. Mol. Psychiatry 2, 393-397 (1997).

10. Ohnishi, T. et al. Spatial expression patterns and biochemical properties distinguish a second myo-inositol monophosphatase IMPA2 from IMPA1. J. Biol. Chem. 282, 637-646 (2007).

11. Jimenez, E. et al. Genetic variability at IMPA2, INPP1 and GSK3beta increases the risk of suicidal behavior in bipolar patients. Eur. Neuropsychopharmacol. $\mathbf{2 3}$ 1452-1462 (2013).

12. Sarkar, S. et al. Lithium induces autophagy by inhibiting inositol monophosphatase. J. Cell Biol. 170, 1101-1111 (2005).

13. Ohnishi, T. et al. Human myo-inositol monophosphatase 2 rescues the nematode thermotaxis mutant $t \mathrm{tx}-7$ more efficiently than IMPA1: functional and evolutionary considerations of the two mammalian myo-inositol monophosphatase genes. J. Neurochem. 124, 685-694 (2013).

14. French, D. et al. Acquired variation outweighs inherited variation in whole genome analysis of methotrexate polyglutamate accumulation in leukemia. Blood 113, 4512-4520 (2009).
15. Lin, Y. F. et al. Dysregulation of the miR-25-IMPA2 axis promotes metastatic progression in clear cell renal cell carcinoma. EBioMedicine 45, 220-230 (2019).

16. Mustafa, D. A. M. et al. T lymphocytes facilitate brain metastasis of breast cancer by inducing Guanylate-Binding Protein 1 expression. Acta Neuropathol. 135, 581-599 (2018)

17. Chen, T. P. et al. Increased expression of SKP2 and phospho-MAPKJERK $1 / 2$ and decreased expression of p27 during tumor progression of cenvical neoplasms. Gynecol. Oncol. 104, 516-523 (2007).

18. $\mathrm{Yu}$, Z. et al. SIX3, a tumor suppressor, inhibits astrocytoma tumorigenesis by transcriptional repression of AURKAVB. J. Hematol. Oncol. 10, 115 (2017).

19. Wang, Z. et al. The D Domain of LRRC4 anchors ERK1/2 in the cytoplasm and competitively inhibits MEK/ERK activation in glioma cells. J. Hematol. Oncol. $\mathbf{9}$ 130 (2016).

20. Wang, Z. Y. et al. Upregulation of the long non-coding RNA AFAP1-AS1 affects the proliferation, invasion and survival of tongue squamous cell carcinoma via the Wnt/beta-catenin signaling pathway. Mol. Cancer 17, 3 (2018).

21. Chandrashekar, D. S. et al. UALCAN: a portal for facilitating tumor subgroup gene expression and survival analyses. Neoplasia 19, 649-658 (2017).

22. Chen, S. Q. et al. Analyzing simultaneous positive expression of EZH2 and P53 protein to improve predictive value in cervical squamous cell carcinoma. Int. J. Gynecol. Cancer 24, 1653-1658 (2014).

23. Chen, Y. et al. INPP4B restrains cell proliferation and metastasis via regulation of the PI3K/AKT/SGK pathway. J. Cell. Mol. Med. 22, 2935-2943 (2018).

24. Xiao, S. et al. Fra-1 is downregulated in cervical cancer tissues and promotes cervical cancer cell apoptosis by p53 signaling pathway in vitro. Int. J. Oncol. 46, 1677-1684 (2015)

25. Yadav, S. S. et al. Epigenetic silencing of CXCR4 promotes loss of cell adhesion in cervical cancer. Biomed. Res. Int. 2014, 581403 (2014).

26. Li, J. et al. A promoter polymorphism rs2075824 within IMPA2 gene affecting the transcription activity: possible relationship with schizophrenia. J. Cell. Mol. Med. 21, 658-664 (2017)

27. Gurnett, C. A. \& Hedera, P. New ideas in epilepsy genetics: novel epilepsy genes, copy number alterations, and gene regulation. Arch. Neurol. 64 324-328 (2007).

28. Tomioka, Y. et al. Association between genetic variation in the myo-inositol monophosphatase 2 (IMPA2) gene and age at onset of bipolar disorder. J. Affect. Disord. 232, 229-236 (2018).

29. Yoon, I. S. et al. Altered IMPA2 gene expression and calcium homeostasis in bipolar disorder. Mol. Psychiatry 6, 678-683 (2001).

30. Sarkar, S. \& Rubinsztein, D. C. Inositol and IP3 levels regulate autophagy: biology and therapeutic speculations. Autophagy 2, 132-134 (2006).

31. Sade, Y. et al. IP3 accumulation and/or inositol depletion: two downstream lithium's effects that may mediate its behavioral and cellular changes. Transl. Psychiatry 6, e968 (2016).

32. Cuadrado, A. \& Nebreda, A. R. Mechanisms and functions of p38 MAPK signalling. Biochem. J. 429, 403-417 (2010)

33. Sun, Y. et al. Signaling pathway of MAPK/ERK in cell proliferation, differentiation, migration, senescence and apoptosis. J. Recept. Signal Transduct. Res. 35, 600-604 (2015)

34. Ventura, J. J. et al. p38alpha MAP kinase is essential in lung stem and progenitor cell proliferation and differentiation. Nat. Genet. 39, 750-758 (2007).

35. $\mathrm{Hu}, \mathrm{X}$. et al. Polypeptide fraction from Arca subcrenata induces apoptosis and G2/M phase arrest in HeLa cells via ROS-mediated MAPKs pathways. Evid. Based Complement. Alternat. Med. 2015, 930249 (2015).

36. Tan, G. X. et al. PP-22 promotes autophagy and apoptosis in the nasopharyngeal carcinoma cell line CNE-2 by inducing endoplasmic reticulum stress, downregulating STAT3 signaling, and modulating the MAPK pathway. J. Cell. Physiol. https://doi.org/10.1002/jcp.27076 (2018).

37. Chiu, C. C. et al. p38 MAPK and NF-kappaB pathways are involved in naphtho [1,2-b] furan-4,5-dione induced anti-proliferation and apoptosis of human hepatoma cells. Cancer Lett. 295, 92-99 (2010).

38. Li, Z. H., Li, L., Kang, L. P. \& Wang, Y. MicroRNA-92a promotes tumor growth and suppresses immune function through activation of MAPK/ERK signaling pathway by inhibiting PTEN in mice bearing U14 cenvical cancer. Cancer Med. https://doi.org/10.1002/cam4.1329 (2018).

39. Sui, X. et al. p38 and JNK MAPK pathways control the balance of apoptosis and autophagy in response to chemotherapeutic agents. Cancer Lett. 344, 174-179 (2014)

40. Hommes, D. W., Peppelenbosch, M. P. \& van Deventer, S. J. Mitogen activated protein (MAP) kinase signal transduction pathways and novel antiinflammatory targets. Gut 52, 144-151 (2003). 
41. Potocnjak, I., Gobin, I. \& Domitrovic, R. Carvacrol induces cytotoxicity in human cervical cancer cells but causes cisplatin resistance: involvement of MEK-ERK activation. Phytother. Res. 32, 1090-1097 (2018).

42. Vazhappilly, C. G. et al. Inhibition of SHP2 by new compounds induces differential effects on RAS/RAF/ERK and PI3K/AKT pathways in different cancer cell types. Investig. New Drugs https://doi.org/10.1007/s10637-018-0626-5 (2018).

43. Zheng, H. Y., Shen, F. J., Tong, Y. Q. \& Li, Y. PP2A inhibits cervical cancer cell migration by dephosphorylation of p-JNK, p-p38 and the p-ERK/MAPK signaling pathway. Curr. Med. Sci. 38, 115-123 (2018).

44. Liu, X., Yang, Q., Yan, J., Zhang, X. \& Zheng, M. LncRNA MNX1-AS1 promotes the progression of cervical cancer through activating MAPK pathway. J. Cell. Biochem. https://doi.org/10.1002/jcb.27712 (2018).

45. Sun, Q., Liang, Y., Zhang, T., Wang, K. \& Yang, X. ER-alpha36 mediates estrogenstimulated MAPK/ERK activation and regulates migration, invasion, proliferation in cervical cancer cells. Biochem. Biophys. Res. Commun. 487, 625-632 (2017).
46. Wang, K. \& Zhu, Y. Dexmedetomidine protects against oxygen-glucose deprivation/reoxygenation injury-induced apoptosis via the p38 MAPK/ERK signalling pathway. J. Int. Med. Res. 46, 675-686 (2018).

47. Chen, J. C., Huang, C., Lee, I. N., Wu, Y. P. \& Tang, C. H. Amphiregulin enhances cell migration and resistance to doxorubicin in chondrosarcoma cells through the MAPK pathway. Mol. Carcinog. 57, 1816-1824 (2018).

48. Xiong, W. et al. DAPK1-ERK signal mediates oxygen glucose deprivation reperfusion induced apoptosis in mouse N2a cells. J. Neurol. Sci. 387, 210-219 (2018).

49. Zong, D. et al. Notch1 regulates endothelial apoptosis via the ERK pathway in chronic obstructive pulmonary disease. Am. J. Physiol. Cell Physiol. 315 C330-C340 (2018).

50. Nass, J. \& Efferth, T. Insights into apoptotic proteins in chemotherapy: quantification techniques and informing therapy choice. Expert Rev. Proteomics 15, 413-429 (2018) 